\title{
Unidade e multiplicidade do tempo: uma abordagem transdisciplinar
}

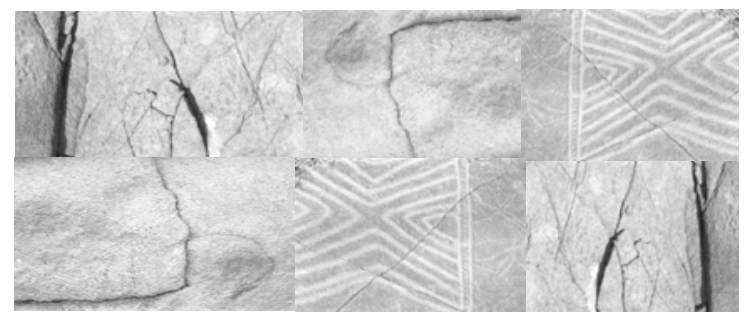

Alfredo Pereira Júnior ${ }^{1}$ Ivan Amaral Guerrini ${ }^{2}$

PEREIRA JR., A.; GUERRINI, I. A. The unicity and multiplicity of time: a transdisciplinary approach, Interface Comunic., Saúde, Educ., v.8, n.15, p.247-56, mar/ago 2004.

This article seeks to discuss time within the context of health sciences, where physical, biological, psychological and sociological factors interact. Whereas in our perception of the world and of ourselves time shows itself from many angles, in classical physics, according to the Newtonian model, physical time was conceived as absolute, unilinear, homogeneous and observer-independent. With the theory of relativity and the study of complex systems, a new concept of time appeared in physics, namely, fractal time, which is more compatible with psychological and sociological approaches. From this point of view, the life experience of a person and the respective processes of health construction involve a multiplicity of coexisting temporalities, organized in a coherent pattern of self-similarity. A rupture in this coherent pattern leads to the appearance of illness. The article suggests that a more suitable approach to sickness ought to take into account, as a reference for healthcare professionals, the concept of fractal time, causing the patient to become better attuned to the complexity of nature and, consequently, to himself.

KEY-WORDS: Time; temporality; health-disease process; relativity; fractal; transdisciplinarity.

Procurou-se, neste trabalho, pensar o tempo no contexto das ciências da saúde, no qual se entrelaçam aspectos físicos, biológicos, psicológicos e sociológicos. Enquanto em nossa percepção do mundo e de nós mesmos o tempo se apresenta sob muitas facetas, na física clássica, conforme o modelo newtoniano, assumia-se a existência de um tempo absoluto, unilinear, homogêneo e independente do observador. Com a teoria da relatividade e o estudo dos sistemas complexos, um novo conceito de tempo apresenta-se na física: o tempo fractal, o qual possibilita maior compatibilidade com as abordagens psicológicas e sociológicas. Nesta perspectiva, a experiência de vida de uma pessoa, e seus respectivos processos de construção da saúde, envolveria uma multiplicidade de tempos, que coexistem e se organizam segundo um padrão coerente de auto-similaridade. Uma quebra desse padrão estaria correlacionada com a ocorrência da doença. Sugere-se que uma abordagem mais adequada do adoecimento deveria levar em conta, como referência para o profissional de saúde, o conceito de tempo fractal, possibilitando maior sintonia do paciente com a complexidade da natureza e, por conseguinte, consigo mesmo.

PALAVRAS-CHAVE: Tempo; processo saúde-doença; relatividade; fractal; transdisciplinaridade.

\footnotetext{
${ }^{1}$ Professor Adjunto, Departamento de Educação, Instituto de Biociências, Unesp, Botucatu, SP. <apj@ibb.unesp.br>

2 Professor Titular, Departamento de Física e Biofísica, Instituto de Biociências, Unesp, Botucatu, SP. <guerrini@ibb.unesp.br>
}

${ }^{1}$ Departamento de Educação

Instituto de Biociências, Unesp

Distrito de Rubião Jr., s/no Botucatu, SP

18.618-000 
PEREIRA JR., A.; GUERRINI, I. A.

\section{Introdução}

Estudos na área de saúde lidam com sistemas complexos, envolvendo desde a escala do organismo individual até grandes populações. Por razões decorrentes do próprio método científico moderno, muitas vezes a abordagem dos fenômenos se faz em termos de uma metodologia reducionista, quando se buscam causas simples, lineares e estanques para processos suscetíveis de múltiplas determinações - de ordem física, biológica, psicológica e social.

Uma forma de se evitar a perda do entendimento da dinâmica dos sistemas complexos é o enfoque de temas integradores, como é o caso do tempo. Não que se considere que o tempo por si só tenha influência sobre os processos que determinam a saúde ou a doença, mas sim que constitua uma dimensão fundamental destes processos, cujo enfoque pode contribuir para iluminar aspectos deixados em segundo plano pela abordagem reducionista.

Por exemplo, a dimensão temporal leva-nos a considerar a existência de ciclos epidêmicos, ao invés de simples processos de emergência e extinção de agentes nocivos. Da mesma forma, pode-se compreender a "terceira idade" não só em termos dos aspectos biológicos degenerativos, mas também como uma época na existência temporal do indivíduo na qual se abre a possibilidade de novas experiências saudáveis.

Por outro lado, sendo o tempo uma dimensão fundamental dos processos humanos, não é de se surpreender que nas mais diversas patologias se observem distúrbios de ordem temporal. Por exemplo, o stress acentuado $e$ persistente pode levar a perturbações no ritmo circadiano, e diversas psicopatologias são acompanhadas de sinais de desorientação temporal.

Tendo em vista o envolvimento do tempo nos processos que se desenrolam na área de Saúde, a questão que se levanta é: será que se pode referir todos eles a um único tempo absoluto e linear, ou seria necessário trabalhar com uma multiplicidade de tempos que se relacionam de forma complexa? Neste trabalho, pretende-se mostrar que a segunda alternativa permite um melhor entendimento do processo saúde-doença. Apresenta-se, pois, a hipótese de que uma vida saudável possibilita a organização dos diversos aspectos temporais em termos de um padrão coerente de autosimilaridade, ou seja, em um tempo fractal, ao passo que processos de doença se caracterizariam por uma desorganização temporal.

Ressalvamos que a aplicação de modelos complexos de tempos ou temporalidades múltiplos no campo da saúde mental já tem sido objeto de discussão no âmbito da psicoanálise (por exemplo, ver Katz, 1996).

\section{O modelo "normal" e seus limites}

A dimensão do tempo tem sido considerada um dos principais aspectos da subjetividade, sendo chamada por Kant (1983) de "o sentido interno". Este sentido ordenaria todas as experiências do sujeito em termos de uma sucessão de eventos. As propriedades dessa sucessão de eventos têm sido estudadas tanto em nível fenomenológico introspectivo (Husserl, 1964; comentado por Pereira Jr., 1990) quanto em nível da filosofia das ciências da natureza. 
Assume-se, em pesquisas sobre o "tempo objetivo" na filosofia da física (vide Newton-Smith, 1980; Pereira Jr., 1997), a existência de um modelo "normal", com as seguintes características:

a) o tempo tem uma "direção", ou seja, uma ordem temporal caracteriza-se por estabelecer uma ordem de sucessão entre os eventos, sejam estes eventos subjetivos ou objetivos;

b) o tempo possui "transitividade", ou seja, se um evento A tem uma relação temporal com um evento $B$ e esse evento $B$ tem uma relação com um evento $C$, então A também tem uma relação temporal com $C$;

c) o tempo é "assimétrico", ou seja, existe uma relação de anterioridade entre os eventos tal que, se $A$ é anterior a $B$, então $B$ não é anterior a $A$;

d) a métrica do tempo é "homogênea" ou "linear", ou seja, o tempo se reparte em unidades idênticas, que se repetem de modo uniforme.

Seria o modelo "normal" acima plenamente adequado para o estudo da experiencia temporal humana?

Da perspectiva da psicologia, o modelo "normal" apresenta, de início, duas grandes limitações: a suposição de que o tempo seria unidimensional e a clássica separação newtoniana entre tempo relativo e absoluto.

$\mathrm{Na}$ temporalidade da experiência humana, encontram-se três dimensões dignas de consideração:

a) a experiência individual subjetiva, incluindo a dinâmica do inconsciente $e$ o fluxo consciente, que compõe uma totalidade que se expressa parcialmente nos relatos lingüísticos e produção significativa do sujeito;

b) a construção social do tempo, que se expressa em obras culturais e instrumentos de registro, consideradas como construções coletivas que constrangem as experiências individuais (Elias, 1998); e

c) a temporalidade que perpassa o ambiente físico do sujeito na forma de transformações irreversíveis (representadas na física pelo aumento da

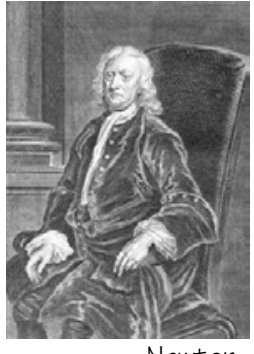

Newton

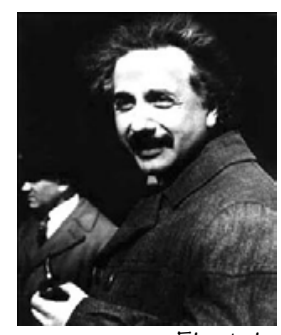

Einstein entropia) e da sucessão causal entre os eventos percebidos.

Seriam estas três dimensões redutíveis a uma única? Com certeza não, razão pela qual o próprio Isaac Newton propôs a distinção entre o tempo absoluto, que serviria como padrão de referência para a física, e os tempos relativos, que poderiam abranger as formas subjetivas e sociais do tempo. Entretanto, a distinção entre um tempo físico único e uma multiplicidade de tempos subjetivos caiu por terra no contexto da própria física, quando Einstein mostrou que a métrica temporal está vinculada ao estado de movimento dos corpos relativamente à velocidade da luz no vácuo. Deste modo, o próprio tempo físico seria relativo, e, portanto, múltiplo.

Para um melhor entendimento do tempo, torna-se necessário repensar a relação entre a temporalidade subjetiva humana, sua reelaboração social $e$ o tempo físico, este último concebido como relativo e múltiplo. Como estas três dimensões se harmonizam, e/ou como conflitam entre si? Quais seriam as conseqüências, para a saúde mental, da harmonia e/ou do conflito entre tais dimensões da experiência temporal?

\section{A multiplicidade temporal na existência humana}

Quando se considera a experiência temporal humana em sua complexidade, algumas importantes questões emergem: 
a) como se relacionam as três dimensões acima citadas? Existe uma estrutura universal do tempo perpassando todas elas?

b) como seria a percepção do tempo nas psicopatologias, uma vez que elas afetam o tempo da experiência subjetiva, mas não necessariamente o tempo físico e social?

c) seriam as alterações do tempo subjetivo, observáveis na psicopatologia, identificáveis relativamente ao modelo "normal" acima delineado?

Começando a discussão pela última questão, entende-se que violações flagrantes do modelo "normal" seriam, por exemplo, se eventos ocorridos no passado "acontecessem de novo", ou ainda se eventos imaginados do futuro fossem vividos como ocorrendo no presente. Neste caso, estaria havendo uma alteração relativamente à característica de assimetria temporal. Se uma pessoa não tem noção de datas, poderia haver uma perda da noção de direção temporal. Se os eventos do presente aparecem como isolados do passado e do futuro, sem uma dimensão de continuidade, haveria uma alteração da característica de transitividade. Se alguns instantes ou intervalos temporais psicologicamente parecem ter maior duração que outros de mesmo tamanho, haveria a perda da característica de homogeneidade ou linearidade temporal.

Tais raciocínios seriam o resultado da aplicação direta do modelo "normal" a situações aparentemente "anormais". Por outro lado, se o modelo dito "normal" não constitui um modelo universal da experiência humana, outras possibilidades de interpretação emergem.

Considere-se a possibilidade de um outro tipo de modelagem do tempo, no qual coexistam várias dimensões temporais na experiência de vida de uma pessoa, cada uma delas possuindo uma métrica (ou "ritmo") própria, por exemplo:

- tempos biológicos, como o ritmo circadiano (períodos intercalados de vigília e sono), escalonamento diário da alimentação, atividade física etc;

- tempos ligados ao trabalho, como uma jornada diária de oito horas, repouso nos fins de semana, férias anuais etc;

- tempos ligados à mudança do clima, como as estações do ano;

- tempos de maturação psicológica, como períodos de adolescência, casamento, cuidados com filhos, moradia etc.

Considerando-se todas essas dimensões, que coexistem e se interrelacionam na vida de uma pessoa, pode-se avaliar que as alterações em uma delas não necessariamente implicam alterações equivalentes nas demais. Ao contrário, podem ocorrer processos de compensação, tal que, por exemplo, uma alteração climática seja compensada por mudanças nas atividades sociais, como acontece no "horário de verão" instituído em alguns países. Neste caso, o tempo social é "adiantado" e depois "atrasado", violando o modelo "normal", sem que com isso haja qualquer aberração.

$\mathrm{Na}$ área de saúde mental, acredita-se tradicionalmente (Minkowski, 1933), como uma resposta à segunda questão colocada acima, que a esquizofrenia envolva uma alteração na percepção do tempo, porém observamos que isso pode ser um efeito do contexto. Por exemplo, uma pessoa internada em um hospital onde não há relógios para serem consultados ou atividades a serem cumpridas dentro de um horário, está em 
um contexto que favorece uma alteração na sua percepção do tempo. De certo modo, a desorientação temporal que aí se instala encontra-se adaptada ao contexto em que se desenvolve a experiência temporal. Em um contexto diferente, esses pacientes poderiam re-sintonizar sua percepção do tempo com os padrões vigentes na sociedade em que vivem.

Portanto, ao se considerar a complexidade da existência humana, o foco passa a ser a forma como as diversas dimensões temporais se relacionam, ou seja, se a diversidade de tempos é integrada de modo harmonioso ou conflituoso. No exemplo citado, como a alteração no tempo social se faz de modo harmonioso com as mudanças do clima (isto é, do "tempo" no sentido meteorológico), pode-se conseguir uma integração mais saudável dos diversos aspectos temporais envolvidos na vida das pessoas. Por outro lado, se sistematicamente ocorrem conflitos, por exemplo, entre uma jornada de trabalho noturna e o ritmo circadiano, instaura-se uma configuração de fatores que pode aumentar a propensão a certas doenças.

Uma relação harmoniosa entre os diversos tempos que ocorrem na vida de uma pessoa pode ser comparada a uma orquestra bem ensaiada, na qual as diversas vozes musicais se integram coerentemente no tempo. Por exemplo, em uma execução em compasso quaternário, alguns instrumentos (A) podem executar dezesseis notas por compasso, enquanto outros (B) executam quatro notas, e outros (C) executam apenas uma nota. Essa estrutura musical pode apresentar a propriedade de auto-similaridade, se o padrão formado pelos instrumentos A e B for similar ao padrão formado pelos instrumentos $B$ e $C$, ou seja, para cada nota executada por $B$ há a execução de quatro notas por $A$, e para cada nota executada por $C$ também há a execução de quatro notas por $\mathrm{B}$. Mesmo que os instrumentos $\mathrm{C}$, por exemplo, alterem seu padrão temporal, a estrutura auto-similar pode se manter, caso os demais instrumentos também alterem seus padrões de modo coerente.

A auto-similaridade funcional, que aqui nos interessa, e constitui uma possível resposta à primeira questão colocada acima, diz respeito à similaridade entre os padrões de atividade que se formam nas diversas escalas espaciais e/ou temporais que compõem a dinâmica total de um sistema. Deste modo, a universalidade da experiência temporal se manifestaria como presença de auto-similaridade, e não como um tempo absoluto. No exemplo acima, se a orquestra passar para um compasso ternário, quando para cada nota executada pelos instrumentos $B$ há três notas executadas por $A$, e para cada nota executada por $C$ há três notas executadas por $\mathrm{B}$, a auto-similaridade funcional se mantém.

A característica de auto-similaridade pode ser visualizada em uma figura de M. Escher (Fig. 1), que foi elaborada independentemente de tais desenvolvimentos da ciência, mas antecipa genialmente o conceito, possivelmente devido à apurada intuição do artista.

A Figura 1 pode ser interpretada em termos da estrutura da experiência temporal aqui referida, associando-se aos símbolos os diversos aspectos dessa experiência, como por exemplo: 
PEREIRA JR., A.; GUERRINI, I. A.

$A 1+B 1:$ período de 24 horas

Al: período de vigília

B1: período de sono

C1: período de trabalho

E1: período de lazer

D1: período de sono sem sonhos

$\mathrm{Fl}$ : período de sono com sonhos,

e daí por diante, subdividindo-se os períodos maiores em períodos menores, os quais se inserem de modo coerente dentro dos primeiros.

Figura 1 - llustração de Estrutura Auto-Similar por meio de desenho de M. Escher: diversas escalas espaço-temporais são representadas, apresentando um padrão de auto-similaridade.O triângulo com um círculo inserido, ao topo, pode simbolizar a integração coerente de toda a estrutura subjacente.

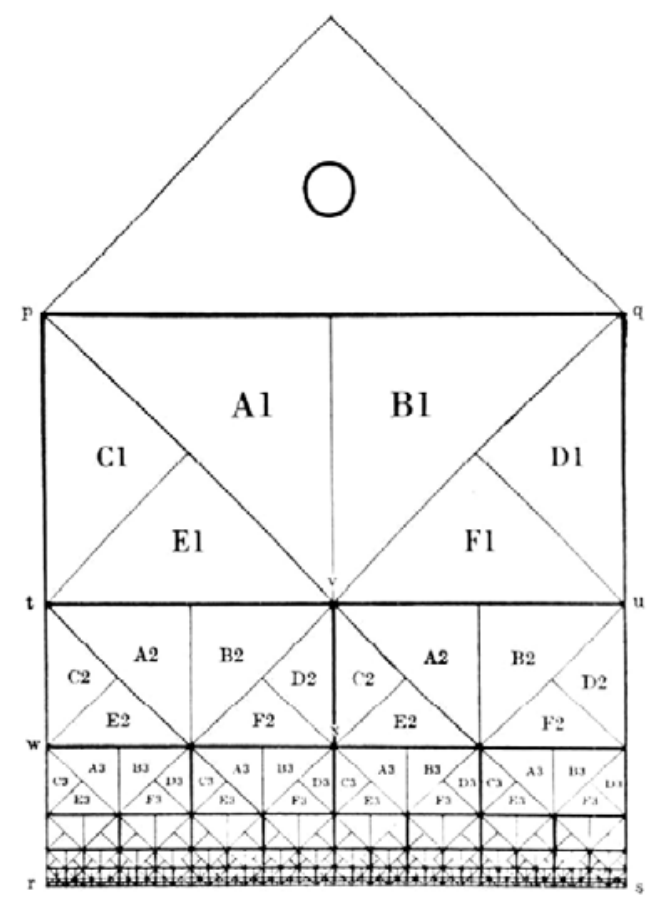

Como ilustração desta proposta, citamos um estudo interessante sobre a sincronização dos ciclos biológicos humanos (Cherry, 2002), particularmente o equilíbrio dinâmico da produção dos neuromoduladores serotonina e melatonina, com a radiação global da Terra e de sua camada mais baixa da ionosfera, conhecida como Ressonância de Schumann. A falta de ressonância desses sinais (Terra e seres humanos) provocaria distúrbios nas pessoas. Como a freqüência do sinal de Schumann tem aumentado significativamente nas últimas décadas, haveria uma tendência de alteração na percepção do tempo para o ser humano, com conseqüentes desequilíbrios quando a sincronização não é alcançada. 
A unidade na multiplicidade: tempo fractal A partir da Teoria da Relatividade de Einstein, pode-se conceber uma multiplicidade de tempos físicos, considerados como relativos às características (também físicas) dos observadores. Para aquela teoria física, o fator central é a velocidade em que o observador se move. Assim, para observadores em velocidade próxima à da luz no vácuo $(300.000 \mathrm{~km} / \mathrm{s})$, há uma dilatação do tempo, fazendo com que os processos percebidos pelos referenciais estacionários ocorram de forma mais lenta; para velocidades menores, o fator de dilatação do tempo se torna desprezível e a métrica do tempo se aproxima do modelo "normal". Generalizando a concepção de um tempo relativo $e$ múltiplo para a área de saúde, propõe-se introduzir a idéia de que não só o estado de movimento do observador (sujeito) é importante, como na Teoria da Relatividade na física, mas também outros fatores psicobiológicos e ambientais poderiam condicionar a percepção do tempo.

A Teoria dos Fractais também vem introduzir novas possibilidades de compreensão do tempo. Assim, o conceito de "tempo fractal" emerge a partir do conhecimento dos fractais artificiais e naturais, explicados em abrangente literatura desde as primeiras idéias publicadas por Mandelbrot (1983). A idéia central sobre o tempo fractal é semelhante ao espaço fractal, cujo exemplo típico é o movimento browniano (random walk). Alguns autores, em estudos recentes sobre tempo e espaço na ciência e na vida (Briggs \& Peat, 2000), aventam a possibilidade de se ter um tempo natural também com essa tortuosidade e complexidade, já que isso é possível de se encontrar no espaço. Segundo esses autores, as intuições, por exemplo, ocorreriam nesses intervalos de tempo fractal, ou seja, na interface de diferentes níveis dimensionais, como sugere a abordagem transdisciplinar detalhada por Nicolescu (1999).

Assim, argumentam Briggs \& Peat (2000), se na Teoria dos Fractais o modelo linear de espaço foi trocado pelo irregular, este mais abrangente $e$ trazendo padrões escondidos como no caso do Movimento Browniano (random walk), por que o tempo não poderia ser visto assim também? Neste ponto, o conceito de espaço-tempo de Einstein poderia auxiliar, já que a entidade única fundamental que permanece seria o espaço-tempo e não mais espaço e tempo como "entidades" separadas. Daí resultam as chamadas "dobras do tempo", próprias de um tempo fractal, em que as linearidades temporais deixam de existir, numa visão mais abrangente e transdisciplinar da natureza, ficando as regiões de linearidade como uma aproximação válida somente em casos limites.

Um outro aspecto a ser abordado em relação à multiplicidade de tempos é a questão da definição e representação da "ordem" na ciência e na sociedade, principalmente no que se refere aos conceitos de espaço e tempo (Bohm \& Peat, 2000). Assim como uma linha reta pode ser vista como a integração de infinitos segmentos de reta colocados em sequência linear, o mesmo ocorreria com relação ao tempo. O tempo linear, na verdade, refletiria uma idéia classicamente arquetípica de soma de infinitos e pequenos intervalos de tempo, todos iguais, lineares e possíveis de serem medidos em relógios comuns.

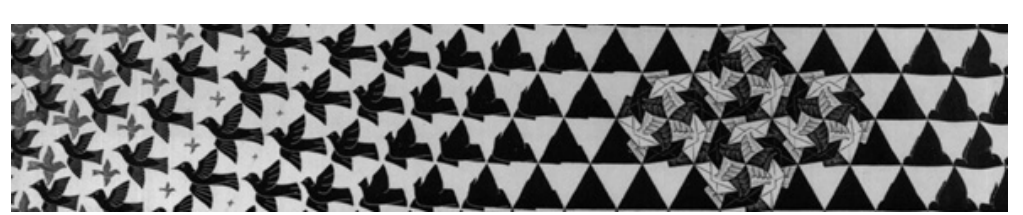

Porém, como no caso dos segmentos de reta, a união dos intervalos poderia se dar, por exemplo, com cada segmento fazendo um ângulo diferente de zero e de valor 
PEREIRA JR., A.; GUERRINI, I. A.

constante com o segmento anterior (segundo grau de ordem ou ordenação), o que permitiria a construção de um conjunto de segmentos formando diferentes figuras, conforme o ângulo utilizado. Além disso, se sempre que se adicionar a um segmento houver uma variação igual desse ângulo no mesmo plano, para cada segmento incorporado (terceiro grau de ordem ou ordenação), a figura se altera completamente. Se sair do plano inicial, formando ângulos com o outro eixo ortogonal, outras figuras mais complexas se formam, com outros graus de ordem. Se a razão do tamanho de um segmento para seu anterior for constante, mas diferente da unidade, ainda outros graus de ordem aparecem em cada caso citado.

Em qualquer formação natural, o desafio do cientista, conforme essa visão, seria encontrar a "ordem" do sistema, ou seja, aquele grau de ordem ou aquela ordenação embutida no sistema que traria maior e melhor informação sobre o mesmo. Mandelbrot (1983) descobriu isso, por exemplo, nas irregularidades da linha costeira da Noruega, identificando nelas a auto-similaridade típica dos fractais, com um elevado grau de ordem. Posteriormente, os cientistas descobriram essa "ordem escondida" com diferentes graus em árvores, folhas, nuvens, solos, formações rochosas e em inúmeras outras formações naturais, em que, por conseqüência, o macro se refletia no micro.

É essa busca da ordem escondida nos padrões fractais que levou Prigogine (2000) a questionar o tempo linear e reversível da física clássica. Os problemas de Física Clássica, ministrados para alunos de ensino médio e universidades, ainda hoje adotam uma visão exclusivamente newtoniana. É a reversibilidade do tempo implícita nas leis de Newton que a definiram sempre como tendo um caráter eterno, motivo pelo qual se inseriu muito bem na religiosidade do século XVII e seguintes. A questão do grau de ordem do tempo, definindo o tempo fractal com suas dobras, é um passo além da proposta de Prigogine, muito embora tenha aí suas raízes profundas. À semelhança do que ocorre no espaço medido num movimento randômico, as dobras do tempo permitiriam uma concepção de tempo bem distinta da clássica, em que o "comprimento" final do tempo pode tender ao infinito, dependendo do grau de ordem envolvido.

Portanto, entende-se que uma abordagem mais adequada da experiência do tempo deveria se reportar ao conceito de tempo fractal, que se caracteriza por duas características principais, aqui enfocadas:

a) a existência da auto-similaridade;

b) irregularidades que coexistem com a auto-similaridade.

Ao invés de se oporem, a auto-similaridade emerge da própria irregularidade, ou melhor, de uma irregularidade complexa, na qual diversos padrões coexistentes interagem e se compensam, gerando um certo grau de ordem. Assim, os conceitos de ordem e desordem deixam de ser considerados como contraditórios e passam a ser considerados como complementares na descrição da complexidade dos fenômenos naturais. É a "ordem dentro da desordem" a que se referia Mandelbrot (1983).

Para uma melhor compreensão da complexidade, é importante o conceito de transdisciplinaridade em ciência (Nicolescu, 1999), o que exige uma abertura para novas dimensões do conhecimento humano. Nesse sentido, uma das grandes dificuldades é uma forte tendência do cientista clássico em se segurar naquilo que lhe é familiar (Bohm \& Peat, 2000), defendendo-se, por todas as 
formas, dos conceitos novos que o desafiam e parecem querer desestabilizá-lo. Para esses cientistas, a ciência é um conjunto muito grande de normas e leis finitas que, um dia totalmente esclarecido, chegará à noção absoluta da verdade. De forma diferente, a transdisciplinaridade parte de um conceito de ciência como sistema aberto à transcendência para diferentes dimensões.

Desse ponto de vista, Einstein foi clássico ao atribuir aos fenômenos estranhos da Física Quântica as famosas "variáveis ocultas" que, uma vez vindas à luz, os explicariam sem precisar recorrer às mudanças inquietantes que se processavam nas leis físicas do mundo quântico. Bohm \& Peat (2000) salientam que é exatamente aceitando o tempo não-linear que se abrem possibilidades para se compreender a criatividade do ser humano em sintonia com a natureza. Essa talvez também seja uma chave para a compreensão do conceito e da vivência temporal na área de saúde, podendo facilitar o tratamento de várias doenças, principalmente em questões de saúde mental.

Se a não-linearidade temporal favorece a criatividade, os graus de liberdade implícitos nos comportamentos criativos não poderiam ser sufocados em pessoas que procuram a saúde mental. Portanto, os modelos terapêuticos e de reabilitação poderiam compatibilizar a indução de novos padrões cíclicos na experiência temporal dos pacientes (compondo uma estrutura auto-similar coerente e auto-sustentável), com a presença de graus de liberdade comportamental que possibilitem a realização da pessoa enquanto sujeito criativo. Estes graus de liberdade podem ser induzidos, por exemplo, por atividades de arte-terapia.

Na mesma linha de raciocínio, Kyriasis (2003) salienta que o fenômeno do envelhecimento humano, com as doenças a ele relacionadas, se correlaciona com uma perda da complexidade do organismo humano. Esse autor salienta que a Teoria do Caos tem desafiado a visão clássica dos regimes farmacológicos, assegurando que para se atingir benefícios máximos com idosos, deve-se fornecer medicação com intervalos de tempo irregulares e em doses constantemente alteradas. Para ele, a natureza prefere a irregularidade à monotonia, o que nos leva a questionar a concepção linear do tempo implícita nos atuais paradigmas terapêuticos. De uma perspectiva mais ampla, tal irregularidade deve ser planejada de acordo com o padrão de auto-similaridade peculiar à experiência temporal de cada pessoa, combinando-se dessa maneira esforços para a recuperação da coerência temporal das funções fisiológicas, com um certo grau de "irregularidade coerente" que incite a manifestação de todos os possíveis recursos de defesa do organismo.

\section{Conclusão}

As idéias aqui discutidas permitem rever os conceitos clássicos do tempo, introduzindo novas concepções a partir das teorias da Relatividade e de Sistemas Dinâmicos Complexos. A sintonia entre as várias dimensões do tempo na vida humana é proposta em termos da formação de um padrão de auto-similaridade coerente, contendo irregularidades intrínsecas, o tempo fractal. Sugerimos que a concepção do tempo como fractal poderia auxiliar no enfrentamento das mais variadas enfermidades, particularmente no caso de doenças mentais. Assim, uma abertura dos profissionais de saúde para uma compreensão mais abrangente do tempo, na etiologia e tratamento das doenças, poderia conduzir a um melhor 
PEREIRA JR., A.; GUERRINI, I. A.

entendimento do processo saúde-doença $e$ inspirar novas pesquisas em áreas que lidam com a complexidade da experiência humana.

\section{Referências}

BOHM, D; PEAT, F.D. Science, order, and creativity. 2.ed. London: Routledge, 2000.

BRIGGS, J.; PEAT, F.D. Sabedoria do caos. Rio de Janeiro: Ed. Campus, 2000.

CHERRY, N. Schumann resonances and their possible biophysical effects. Nat. Hasards, n.26, p.219, 2002.

ELIAS, N. Sobre o tempo. Trad. V. Ribeiro. Rio de Janeiro: Zahar, 1998.

HUSSERL, E. Leçons pour une Phénoménologie de la conscience intime du temps. Trad. Henri Dussort. Paris: Presses Universitaires de France, 1964.

KANT, E. Crítica da razão pura. Trad. V. Rohden, U. Moosburger. São Paulo: Abril Cultural, 1983. (Col. Os Pensadores, v.25).

KATZ, C.S. (Org.) Temporalidade e psicanálise. Petrópolis: Vozes, 1996.

KYRIASIS, M. Practical applications of chaos theory to the modulation of human ageing: nature prefer chaos to regularity. Biogerontology, v.4, n.2, p.75-90, 2003.

MANDELBROT, B. B. The fractal geometry of nature. New York: W. H. Freeman and Company, 1983.

MINKOWSKI, E. Le temps vécu. Paris: Artrey, 1933.

NEWTON-SMITH, W. The structure of time. Boston: Routledge and Kegan Paul, 1980.

NICOLESCU, B. Manifesto da transdisciplinaridade. São Paulo: Triom, 1999.

PEREIRA JR., A. A percepção do tempo em Husserl. Trans/Form/Ação, n.13, p.73-83, 1990.

PEREIRA JR., A. Irreversibilidade física e ordem temporal na tradição boltzmanniana. São Paulo: Ed. Unesp/Fapesp, 1997.

PRIGOGINE, I. As leis do caos. São Paulo: Ed. Unesp, 2000.

PEREIRA JR., A.; GUERRINI, I. A. Unidad y multiplicidad del tiempo: un abordaje transdisciplinar, Interface - Comunic., Saúde, Educ., v.8, n.15, p.247-56, mar/ago 2004.

En este trabajo, se intentó pensar el tiempo en el contexto de las ciencias de la salud, en el cual se entrelazan aspectos físicos, biológicos, psicológicos y sociológicos. Mientras en nuestra percepción del mundo y de nosotros mismos el tiempo se presenta bajo muchas facetas, en la física clásica, conforme el modelo newtoniano, se asumía la existencia de un tiempo absoluto, unilineal, homogéneo e independiente del observador. Con la teoría de la relatividad y el estudio de los sistemas complejos, un nuevo concepto de tiempo se presenta en la física: el tiempo fractal, el cual posibilita una mayor compatibilidad con los abordajes psicológicos y sociológicos. Desde esta perspectiva, la experiencia de vida de una persona, y sus respectivos procesos de construcción de la salud, involucraría una multiplicidad de tiempos, que coexisten y se organizan según un patrón coherente de autosimilaridad. Una quiebra de ese patrón estaría correlacionada con la ocurrencia de la enfermedad. Se sugiere que un abordaje más adecuado de la enfermedad debería considerar, como referencia para el profesional de salud, el concepto de tiempo fractal, posibilitando mayor sintonía del paciente con la complejidad de la naturaleza $y$, por consiguiente, consigo mismo.

PALABRAS CLAVE: Tiempo; proceso salud-enfermedad; relatividad; fractal; transdisciplinaridad. 